\title{
Genetic variants in the nucleotide excision repair pathway genes and gastric cancer susceptibility in a southern Chinese population
}

This article was published in the following Dove Press journal: Cancer Management and Research

Jing $\mathrm{He}{ }^{1, *}$ Zhen-Jian Zhuo, ${ }^{2, *}$ Anqi Zhang, ${ }^{3, *}$ Jinhong Zhu, ${ }^{4}$ Rui-Xi Hua, ${ }^{5}$ Wen-Qiong Xue,' Shao-Dan Zhang,' Jiang-Bo Zhang,' Xi-Zhao Li,' Wei-Hua Jia'

'State Key Laboratory of Oncology in South China, Department of Experimental Research, Collaborative Innovation Center for Cancer Medicine, Sun Yat-sen University Cancer Center, Guangzhou, Guangdong, China; ${ }^{2}$ Faculty of Medicine, School of Chinese Medicine, The Chinese University of Hong Kong, Hong Kong, China; ${ }^{3}$ Department of Obstetrics and Gynecology, The Second Affiliated Hospital and Yuying Children's Hospital of Wenzhou Medical University, Wenzhou, Zhejiang, China; ${ }^{4}$ Molecular Epidemiology Laboratory, Department of Laboratory Medicine, Harbin Medical University Cancer Hospital, Harbin, Heilongjiang, China; ${ }^{5}$ Department of Oncology, The First Affiliated Hospital of Sun Yat-sen University, Guangzhou, Guangdong, China

*These authors contributed equally to this work
Correspondence: Wei-Hua Jia; Jing $\mathrm{He}$ State Key Laboratory of Oncology in South China, Department of Experimental Research, Collaborative Innovation Center for Cancer Medicine, Sun Yat-sen University Cancer Center, 65I Dongfeng Road East, Guangzhou 510060, Guangdong, China Tel +86 $2087342327 ;+862087342410$ Fax +86 208734 3392; +862087342410 Email jiawh@sysucc.org.cn; hejing27@mail. sysu.edu.cn
Background: Potentially functional polymorphisms can modulate protein activities and host's DNA repair capacity, thereby influencing cancer susceptibility. The association of the polymorphisms in the nucleotide excision repair core pathway genes and gastric cancer susceptibility remains largely unknown.

Methods: Here, we systematically analyzed the associations between nine polymorphisms in four key genes (XPA, ERCC1, ERCC2, and ERCC4) in the nucleotide excision repair pathway and gastric cancer risk in a Chinese population including 1142 patients and 1173 controls. Odds ratios (ORs) and 95\% confidence intervals (CIs) were used to estimate the risk associations. Results: We observed that ERCC1 rs2298881 CA variant genotype was associated with an increased gastric cancer risk (CA vs. CC: adjusted OR [AOR] $=1.33,95 \% \mathrm{CI}=1.09-1.62$; dominant model: $\mathrm{AOR}=1.32,95 \% \mathrm{CI}=1.10-1.60)$. However, ERCC1 rs3212986 AA variant genotype was identified as a protective factor for gastric cancer (AA vs. $\mathrm{CC}$ : $\mathrm{AOR}=0.73,95 \% \mathrm{CI}=0.54-0.98$; recessive model: $\mathrm{AOR}=0.72,95 \% \mathrm{CI}=0.54-0.96$ ). Genotype-based $\mathrm{mRNA}$ expression analysis further indicated that the rs 2298881 A allele was associated with decreased ERCC1 mRNA expression. Conclusion: In all, these results indicated that the ERCC1 polymorphisms may affect the risk of gastric cancer in the Chinese Han population.

Keywords: gastric cancer, DNA repair, $N E R$, polymorphism, susceptibility

\section{Introduction}

Gastric cancer, one of the most lethal malignancies, is the fourth most common cancer and the second leading deadly cancer in the world. ${ }^{1,2}$ According to statistics of the National Central Cancer Registry of China, gastric cancer ranks second in both incidence and mortality of cancers in China. ${ }^{3}$ Despite remarkable progress, the current treatments for gastric cancer are still not efficacious with overall 5-year survival rates $<30 \% .{ }^{4}$ One of the main reasons for such a predicament might be that most patients were diagnosed at advanced stages of the disease. ${ }^{5}$ Understanding the underlying mechanisms of gastric cancer initiation and progression may promote biomarker development for early detection of cancer.

Increasing evidence has proven that both environmental and genetic factors contribute to the occurrence and development of gastric cancer. ${ }^{6}$ Helicobacter pylori infection is a well-established risk factor for gastric cancer, affecting $>60 \%$ of all gastric cancer cases. ${ }^{7,8}$ However, not all the $H$. pylori-infected patients finally develop gastric cancer. Many other factors also play roles in gastric carcinogenesis, including micronutrient deficiencies, high body mass index, a high salt or a low fiber diet, over consumption of tobacco or alcohol, as well as genetic risk factors. ${ }^{9-11}$ Increasing numbers of genetic variations have been found to influence susceptibility to gastric cancer in the previous epidemiological studies. ${ }^{12,13}$ 
The integrity and stability of the genome are primarily maintained by DNA repair systems, which include base excision repair, double strand break repair, mismatch repair, and nucleotide excision repair (NER). ${ }^{14,15}$ Among these systems, NER system plays a major role in monitoring and repairing DNA damages caused by exogenous and endogenous factors. ${ }^{16}$ Defects in the NER system might threaten the integrity of genome and thus lead to the development of disease. ${ }^{17}$ It is elucidated that reduced DNA repair capacity is most frequently associated with various human diseases including cancer. ${ }^{18}$ NER process consists of four main steps: damage recognition, damage unwinding, damage incision, and new strand ligation. ${ }^{19}$ There are at least eight key proteins (complementation groups XP-A to G and ERCC1) identified to limit the rate of NER process. ${ }^{20}$ Specifically, XPA and XPC play critical roles in recognizing the DNA damage ${ }^{21,22}$; XPD and XPB are responsible for the process of damage unwinding $^{23,24}$; ERCC1, XPF, and XPG are all essential components for the DNA damage incision..$^{25,26}$

Thus far, several studies have been reported concerning the association between the polymorphisms in the NER pathway genes and the outcomes of gastric cancer. ${ }^{27,28}$ However, the association of these polymorphisms with gastric cancer risk was not fully elucidated. Therefore, the aim of this study was to further identify the association between these polymorphisms and gastric cancer susceptibility. In this study, we systematically analyzed the association between nine potential functional single nucleotide polymorphisms (SNPs) in the NER pathway genes (XPA, $E R C C 1, E R C C 2$, and $E R C C 4$ ) and gastric cancer risk using 1142 patients and 1173 cancer-free controls in a southern Chinese population.

\section{Materials and methods Study population}

This study was approved by the Institutional Review Board of Sun Yat-sen University Cancer Center, Guangzhou, Guangdong. The case group comprised 1142 patients with histologically confirmed gastric cancer enrolled from Sun Yat-sen University Cancer Center from February 2002 to September 2013. The control group consisted of 1173 healthy controls randomly recruited from the same region. ${ }^{29,30}$ Enrollment was restricted to unrelated ethnic Han Chinese population from South China. Detailed information was obtained on all subjects, including demographic characteristics (e.g., age and sex), and lifestyle habits (e.g., smoking habits and alcohol drinking). The classification criteria for smoking status and drinking status were described elsewhere. ${ }^{31}$ Written informed consent was acquired from each participant, accompanying with a donation of $5 \mathrm{~mL}$ of venous blood sample.

\section{Polymorphism selection and genotyping}

The potentially functional polymorphisms of main genes in NER pathway were selected from dbSNP database (http:// www.ncbi.nlm.nih.gov/projects/SNP). Specifically, the following items were set as the selection criteria: 1) located at the $5^{\prime}$ untranslated regions (UTR), upstream promoter region, coding region, and $3^{\prime}$ UTR of genes; 2) the minor allele frequency was $>5 \%$ in Chinese Han populations; 3 ) no obvious linkage between paired SNPs in linkage disequilibrium $\left(\mathrm{R}^{2}<0.8\right)$. We also adopted SNPinfo (http://snpinfo.niehs.nih. gov/snpfunc.htm) to predict the potential functions of those polymorphisms; they could affect the activity of transcription factor binding sites or microRNA binding sites. As a result, the following polymorphisms were included: XPA (rs1800975 $\mathrm{G}>\mathrm{A}, \mathrm{rs} 3176752 \mathrm{C}>\mathrm{A}) ; E R C C 1(\mathrm{rs} 2298881 \mathrm{C}>\mathrm{A}, \mathrm{rs} 11615$ $\mathrm{G}>\mathrm{A}, \mathrm{rs} 3212986 \mathrm{C}>\mathrm{A}) ; E R C C 2(\mathrm{rs} 3810366 \mathrm{C}>\mathrm{G}, \mathrm{rs} 238406$ $\mathrm{G}>\mathrm{T}, \mathrm{rs} 13181 \mathrm{~T}>\mathrm{G})$; and ERCC4 $\mathrm{rs} 2276466 \mathrm{C}>\mathrm{G}$.

DNA was extracted from the blood samples using QIAamp DNA Blood mini kit (QIAGEN Inc, Valencia, CA, USA). Genotyping were performed by the Taqman real-time PCR method on 7900 Sequence Detection System (Applied Biosystems, Foster City, CA, USA), as previously described. ${ }^{31-34}$ For quality control purposes, four duplicate positive controls and four negative controls (without DNA) were used in each of 384-well plates. Moreover, $10 \%$ of the samples were randomly selected to re-genotype. There was $100 \%$ genotype concordance for each polymorphism among duplicates.

\section{Statistical analysis}

First, we adopted goodness-of-fit $\chi^{2}$-test to check whether genotype frequencies of each polymorphism in controls were in Hardy-Weinberg equilibrium (HWE). Then the clinical and demographic characteristics were compared between cases and controls, using the two-sided $\chi^{2}$-test. To investigate the association of the polymorphisms with gastric cancer risk, odds ratios (ORs) and 95\% confidence intervals (CIs) were calculated. Multivariate analysis using unconditional logistic regression model was performed to calculate adjusted ORs (AORs), with adjustment for age, sex, pack-years, smoking and drinking status. Genotype-based mRNA expressions were also conducted as we described previously. ${ }^{31,35}$ All statistical analyses were carried out using version 9.1 SAS software (SAS Institute, Cary, NC, USA). A two-sided $P$-value $<0.05$ was used as a criterion of significance. 


\section{Results}

\section{Population characteristics}

This study consisted of 1142 cases of gastric cancer and 1173 healthy controls, whose individual characteristics are shown in Table S1. With regard to sex, there was no statistically significant difference between cases and controls ( $65.59 \%$ male vs. $67.26 \%$ male, $P=0.393$ ). However, significant differences were observed between cases and controls, regarding age, smoking status, drinking status, and pack-years. Thereafter, these variables were further adjusted for in the subsequent multivariate analyses. Overall, 12.26\% (140), 28.81\% (329), $39.93 \%$ (456), and $19.00 \%$ (217) of patients had TNM stage I, II, III, and IV tumors, according to the 7th Edition of the American Joint Committee on Cancer. ${ }^{36}$

\section{Associations between selected polymorphisms and gastric cancer risk}

The raw data in this paper has been successfully uploaded and locked onto Research Data Deposit with a RDD number of RDDA2018000557. The genotype frequencies of all the selected gene polymorphisms among cases and controls are summarized in Table 1. All observed genotype frequencies among the controls were conformed to the HWE. In the single locus analysis, we observed a significantly increased gastric cancer risk associated with the ERCC1 rs2298881 A variant allele (CA vs. $\mathrm{CC}$ : $\mathrm{AOR}=1.33,95 \% \mathrm{CI}=1.09-1.62$; dominant model: $\mathrm{AOR}=1.32,95 \% \mathrm{CI}=1.10-1.60$; and additive model: $\mathrm{AOR}=1.20,95 \% \mathrm{CI}=1.04-1.38)$. However, ERCC1 rs3212986 A variant allele contributed to decreased

Table I Associations between selected polymorphisms and gastric cancer risk

\begin{tabular}{|c|c|c|c|c|c|c|c|}
\hline Genotypes & Cases $(\mathrm{N}=|| 4 \mid)$ & Controls $(N=1173)$ & $P$-value ${ }^{a}$ & OR $(95 \% \mathrm{CI})$ & $P$-value & AOR (95\% Cl) & $P$-value ${ }^{b}$ \\
\hline \multicolumn{8}{|c|}{ XPA rs $1800975 \mathrm{G}>A$} \\
\hline GG & $296(25.94)$ & $327(27.88)$ & & 1.00 & & 1.00 & \\
\hline GA & $575(50.39)$ & $590(50.30)$ & & I.08 (0.89-I.3। $)$ & 0.458 & I.0I (0.8I-I.26) & 0.954 \\
\hline$A A$ & $270(23.66)$ & $256(21.82)$ & & $1.17(0.92-1.47)$ & 0.197 & $1.05(0.8 \mathrm{I}-1.37)$ & 0.693 \\
\hline Dominant & 845 (74.06) & $846(72.12)$ & 0.294 & $1.10(0.92-1.33)$ & 0.295 & $1.02(0.83-1.26)$ & 0.843 \\
\hline Additive model & & & 0.435 & $1.08(0.96-1.21)$ & 0.197 & $1.03(0.90-1.17)$ & 0.702 \\
\hline Recessive & 871 (76.34) & 917 (78.18) & 0.291 & I.II (0.9I-I.35) & 0.291 & $\mathrm{I} .05(0.84-\mid .3 \mathrm{I})$ & 0.665 \\
\hline \multicolumn{8}{|c|}{$X P A$ rs3 I76752 C>A } \\
\hline $\mathrm{CC}$ & $80 I(70.20)$ & $824(70.25)$ & & 1.00 & & 1.00 & \\
\hline CA & $316(27.70)$ & $318(27.11)$ & & $1.02(0.85-1.23)$ & 0.814 & $1.03(0.84-1.27)$ & 0.760 \\
\hline$A A$ & $24(2.10)$ & $31(2.64)$ & & $0.80(0.46-1.37)$ & 0.410 & $0.92(0.50-1.7 I)$ & 0.794 \\
\hline Dominant & $340(29.80)$ & $349(29.75)$ & 0.981 & $1.00(0.84-1.20)$ & 0.981 & $1.02(0.84-1.25)$ & 0.821 \\
\hline Additive model & & & 0.677 & $0.98(0.84-1.15)$ & 0.818 & $\mathrm{I} .0 \mathrm{I}(0.84-\mid .2 \mathrm{I})$ & 0.908 \\
\hline Recessive & II I7 (97.90) & I I 42 (97.36) & 0.394 & $0.79(0.46-1.36)$ & 0.395 & 0.91 (0.50-1.69) & 0.771 \\
\hline \multicolumn{8}{|c|}{$E R C C I$ rs229888I C>A } \\
\hline $\mathrm{CC}$ & $46 \mathrm{I}(40.40)$ & $540(46.04)$ & & 1.00 & & 1.00 & \\
\hline CA & $548(48.03)$ & $500(42.63)$ & & $1.28(1.08-1.53)$ & 0.005 & $1.33(1.09-1.62)$ & 0.005 \\
\hline AA & $132(11.57)$ & $133(11.34)$ & & $1.16(0.89-1.52)$ & 0.276 & $1.31(0.96-1.78)$ & 0.087 \\
\hline Dominant & $680(59.60)$ & $633(53.96)$ & 0.006 & $1.26(1.07-1.48)$ & 0.006 & $1.32(1.10-1.60)$ & 0.003 \\
\hline Additive model & & & 0.018 & $1.14(1.01-1.29)$ & 0.035 & $1.20(1.04-1.38)$ & 0.010 \\
\hline Recessive & $1009(88.43)$ & $1940(88.66)$ & 0.862 & $\mathrm{I} .02(0.79-1.32)$ & 0.862 & $1.13(0.85-1.5 \mathrm{I})$ & 0.404 \\
\hline \multicolumn{8}{|c|}{$E R C C I \mathrm{rs} I|6| 5 \mathrm{G}>\mathrm{A}$} \\
\hline GG & $594(52.06)$ & $592(50.47)$ & & 1.00 & & 1.00 & \\
\hline GA & 465 (40.75) & $489(41.69)$ & & $0.95(0.80-1.12)$ & 0.537 & $0.94(0.78-1.14)$ & 0.533 \\
\hline$A A$ & $82(7.19)$ & $92(7.84)$ & & $0.89(0.65-1.22)$ & 0.467 & $0.86(0.60-1.22)$ & 0.392 \\
\hline Dominant & $547(47.94)$ & $581(49.53)$ & 0.444 & $0.94(0.80-1.11)$ & 0.444 & $0.93(0.77-1.12)$ & 0.418 \\
\hline Additive model & & & 0.691 & $0.95(0.83-1.08)$ & 0.391 & $0.93(0.81-1.08)$ & 0.344 \\
\hline Recessive & $1059(92.8 \mathrm{I})$ & $1081(92.16)$ & 0.549 & $0.91(0.67-1.24)$ & 0.550 & $0.88(0.62-1.24)$ & 0.468 \\
\hline \multicolumn{8}{|c|}{$E R C C I \mathrm{rs} 3212986 \mathrm{C}>\mathrm{A}$} \\
\hline $\mathrm{CC}$ & $477(4 I .8 I)$ & $478(40.75)$ & & 1.00 & & 1.00 & \\
\hline $\mathrm{CA}$ & 535 (46.89) & $535(45.61)$ & & $1.00(0.84-1.19)$ & 0.981 & $1.02(0.83-1.24)$ & 0.878 \\
\hline AA & $129(\mid 1.31)$ & $160(13.64)$ & & $0.81(0.62-1.05)$ & 0.114 & $0.73(0.54-0.98)$ & 0.037 \\
\hline Dominant & $664(58.19)$ & $695(59.25)$ & 0.606 & $0.96(0.8 \mathrm{I}-1.13)$ & 0.606 & $0.95(0.78-1.14)$ & 0.565 \\
\hline Additive model & & & 0.236 & $0.93(0.82-1.05)$ & 0.227 & $0.90(0.78-1.03)$ & 0.125 \\
\hline Recessive & $1012(88.69)$ & $1013(86.36)$ & 0.090 & $0.81(0.63-1.03)$ & 0.090 & $0.72(0.54-0.96)$ & 0.023 \\
\hline
\end{tabular}


Table I (Continued)

\begin{tabular}{|c|c|c|c|c|c|c|c|}
\hline Genotypes & Cases $(N=1|4|)$ & Controls $(\mathrm{N}=|| 73)$ & $P$-value ${ }^{a}$ & OR $(95 \% \mathrm{Cl})$ & $P$-value & AOR $(95 \% \mathrm{Cl})$ & $P$-value ${ }^{b}$ \\
\hline \multicolumn{8}{|c|}{ ERCC2 rs38I0366 C>G } \\
\hline $\mathrm{CC}$ & $331(29.01)$ & $379(32.31)$ & & 1.00 & & 1.00 & \\
\hline CG & $560(49.08)$ & $554(47.23)$ & & $1.16(0.96-1.40)$ & 0.129 & $1.21(0.98-1.50)$ & 0.079 \\
\hline GG & $250(21.91)$ & $240(20.46)$ & & $1.19(0.95-1.50)$ & 0.134 & $1.20(0.92-1.55)$ & 0.181 \\
\hline Dominant & $810(70.99)$ & $794(67.69)$ & 0.085 & $1.17(0.98-1.39)$ & 0.086 & $1.21(0.99-1.48)$ & 0.067 \\
\hline Additive model & & & 0.219 & $1.10(0.98-1.23)$ & 0.110 & $1.10(0.97-1.26)$ & 0.138 \\
\hline Recessive & 891 (78.09) & $933(79.54)$ & 0.393 & $1.09(0.89-1.33)$ & 0.393 & $1.06(0.85-1.33)$ & 0.598 \\
\hline \multicolumn{8}{|c|}{$E R C C 2$ rs238406 G>T } \\
\hline GG & $296(25.94)$ & $343(29.24)$ & & 1.00 & & 1.00 & \\
\hline GT & $556(48.73)$ & $564(48.08)$ & & $1.14(0.94-1.39)$ & 0.181 & $1.20(0.96-1.49)$ & 0.112 \\
\hline TT & $289(25.33)$ & $266(22.68)$ & & $1.26(1.00-1.58)$ & 0.048 & $1.26(0.97-1.63)$ & 0.081 \\
\hline Dominant & $845(74.06)$ & $830(70.76)$ & 0.076 & $1.18(0.98-1.42)$ & 0.077 & $1.22(0.99-1.50)$ & 0.063 \\
\hline Additive model & & & 0.134 & $1.12(1.00-1.26)$ & 0.046 & $1.12(0.99-1.28)$ & 0.075 \\
\hline Recessive & $852(74.67)$ & $907(77.32)$ & 0.135 & $1.16(0.96-1.40)$ & 0.136 & $1.12(0.90-1.39)$ & 0.295 \\
\hline \multicolumn{8}{|c|}{$E R C C 2 \mathrm{rs}|3| 8 \mid \mathrm{T}>\mathrm{G}$} \\
\hline TT & $971(85.10)$ & $982(83.72)$ & & 1.00 & & 1.00 & \\
\hline TG & $161(14.11)$ & $187(15.94)$ & & $0.87(0.69-1.09)$ & 0.235 & $0.85(0.66-1.10)$ & 0.220 \\
\hline GG & $9(0.79)$ & $4(0.34)$ & & $2.28(0.70-7.4 I)$ & 0.173 & $1.37(0.38-4.99)$ & 0.636 \\
\hline Dominant & $170(14.90)$ & $191(16.28)$ & 0.359 & $0.90(0.72-1.13)$ & 0.360 & $0.87(0.67-1.12)$ & 0.262 \\
\hline Additive model & & & 0.175 & $0.94(0.76-1.16)$ & 0.557 & $0.89(0.70-1.13)$ & 0.335 \\
\hline Recessive & $1132(99.21)$ & $1169(99.66)$ & 0.150 & $2.32(0.71-7.56)$ & 0.162 & $1.40(0.38-5.11)$ & 0.609 \\
\hline \multicolumn{8}{|c|}{$E R C C 4 \mathrm{rs} 2276466 \mathrm{C}>\mathrm{G}$} \\
\hline $\mathrm{CC}$ & $663(58.11)$ & $726(61.89)$ & & 1.00 & & 1.00 & \\
\hline CG & $418(36.63)$ & $383(32.65)$ & & $1.20(1.004-1.42)$ & 0.045 & $1.12(0.92-1.36)$ & 0.272 \\
\hline GG & $60(5.26)$ & $64(5.46)$ & & $1.03(0.71-1.48)$ & 0.889 & $0.96(0.64-1.46)$ & 0.860 \\
\hline Dominant & $478(41.89)$ & $447(38.11)$ & 0.063 & $1.17(0.99-1.38)$ & 0.063 & $1.10(0.91-1.32)$ & 0.348 \\
\hline Additive model & & & 0.130 & $1.11(0.97-1.27)$ & 0.148 & $1.05(0.90-1.23)$ & 0.530 \\
\hline Recessive & 1081 (94.74) & 1109 (94.54) & 0.833 & $0.96(0.67-1.38)$ & 0.833 & $0.93(0.62-1.39)$ & 0.709 \\
\hline
\end{tabular}

Notes: ${ }^{\mathrm{a} C h i-s q u a r e}$ test for genotype distributions between cases and controls. ${ }^{b}$ Adjusted for age, gender, smoking, and drinking status. Bold represents any values with a $95 \% \mathrm{Cl}$ excluding I or $P<0.05$.

Abbreviations: AOR, adjusted odds ratio; OR, odds ratio.

gastric cancer risk (AA vs. $\mathrm{CC}$ : $\mathrm{AOR}=0.73,95 \% \mathrm{CI}=0.54$ 0.98; recessive model: $\mathrm{AOR}=0.72,95 \% \mathrm{CI}=0.54-0.96$ ). There were no significant associations between the rest of all SNPs and gastric cancer risk.

\section{Stratification analysis}

Stratified analysis was performed to further analyze the association of two independent ERCC1 rs $2298881 \mathrm{C}>\mathrm{A}$, rs3212986 C >A polymorphisms and gastric cancer risk by age, sex, smoking status, pack-years, drinking status, tumor sites, and TNM stage (Table 2). The risk association with the ERCC1 rs2298881 CA/AA genotypes remained significant in the following subgroups: males $(\mathrm{AOR}=1.37$, 95\% CI $=1.08-1.73)$, never-smokers $(\mathrm{AOR}=1.40,95 \%$ $\mathrm{CI}=1.09-1.79)$, 0 pack-year $(\mathrm{AOR}=1.40,95 \% \mathrm{CI}=1.09$ 1.79), $\leq 30$ pack-years (AOR $=1.74,95 \% \mathrm{CI}=1.19-2.54)$, never drinkers ( $\mathrm{AOR}=1.36,95 \% \mathrm{CI}=1.09-1.69)$, non-cardia (AOR $=1.31,95 \% \mathrm{CI}=1.08-1.60)$, stage $\mathrm{I} / \mathrm{II}(\mathrm{AOR}=1.42,95 \%$ $\mathrm{CI}=1.11-1.82$ ), and stage III/IV (AOR $=1.28,95 \% \mathrm{CI}=1.03-$ 1.59). Moreover, the $E R C C 1$ rs $3212986 \mathrm{C}>\mathrm{A}$ polymorphism
AA variant significantly reduced gastric cancer risk in the following subgroups: age $\leq 58$ years $(\mathrm{AOR}=0.66,95 \%$ $\mathrm{CI}=0.47-0.93)$, males $(\mathrm{AOR}=0.65,95 \% \mathrm{CI}=0.46-0.92)$, never drinkers (AOR $=0.70,95 \% \mathrm{CI}=0.50-0.98)$, and noncardia $(\mathrm{AOR}=0.72,95 \% \mathrm{CI}=0.53-0.97)$.

We also performed a stratification analysis for the $E R C C 2$ gene rs3810366 $\mathrm{C}>\mathrm{G}$ and $\mathrm{rs} 238406 \mathrm{G}>\mathrm{T}$ polymorphisms (Table 3). Both the rs3810366 (AOR=1.32, 95\% CI=1.04$1.68)$ and rs $238406(\mathrm{AOR}=1.32,95 \% \mathrm{CI}=1.03-1.69)$ polymorphisms conferred gastric cancer susceptibility in never drinkers.

\section{Correlation analysis for ERCCI mRNA expression levels and genotypes}

We further conducted the ERCC1 genotype expression correlation analysis (Table S2), aiming to explore underlying molecular mechanisms. The genotype data for 270 individuals were collected from HapMap. ERCC1 mRNA expression levels of lymphoblastoid cell lines from the same 270 individuals were extracted from SNPexp. We observed that 
Table 2 Stratification analysis of $E R C C I$ gene variant genotypes with gastric cancer risk

\begin{tabular}{|c|c|c|c|c|c|c|c|c|}
\hline \multirow[t]{2}{*}{ Variables } & \multicolumn{2}{|c|}{$\begin{array}{l}\text { rs229888 I } \\
\text { (cases/controls) }\end{array}$} & \multirow[t]{2}{*}{ AOR (95\% CI) } & \multirow[t]{2}{*}{$P$-value ${ }^{a}$} & \multicolumn{2}{|c|}{$\begin{array}{l}\text { rs32 I } 2986 \\
\text { (cases/controls) }\end{array}$} & \multirow[t]{2}{*}{ AOR (95\% CI) } & \multirow[t]{2}{*}{$P$-value ${ }^{a}$} \\
\hline & $\overline{\mathrm{CC}}$ & $\overline{\text { CA/AA }}$ & & & $\overline{C C / C A}$ & AA & & \\
\hline \multicolumn{9}{|c|}{ Median age, years } \\
\hline$\leq 58$ & $250 / 470$ & $348 / 546$ & $1.23(0.99-1.52)$ & 0.062 & $540 / 877$ & $58 / 139$ & $0.66(0.47-0.93)$ & 0.017 \\
\hline$>58$ & $211 / 70$ & $332 / 87$ & $1.29(0.89-1.85)$ & 0.176 & $472 / 136$ & $7 \mid / 21$ & $0.97(0.57-1.65)$ & 0.914 \\
\hline \multicolumn{9}{|l|}{ Gender } \\
\hline Male & $310 / 371$ & $439 / 418$ & $1.37(1.08-1.73)$ & 0.009 & $663 / 677$ & $86 / 112$ & $0.65(0.46-0.92)$ & 0.016 \\
\hline Female & $151 / 169$ & $24 I / 215$ & $1.26(0.92-1.72)$ & 0.159 & $349 / 336$ & $43 / 48$ & $0.90(0.55-1.47)$ & 0.679 \\
\hline \multicolumn{9}{|l|}{ Smoking status } \\
\hline Never & $298 / 305$ & $436 / 357$ & 1.40 (I.09-I.79) & 0.008 & $644 / 571$ & $90 / 91$ & $0.76(0.53-1.10)$ & 0.143 \\
\hline Ever & $163 / 235$ & $244 / 276$ & $1.26(0.93-1.70)$ & 0.134 & $368 / 442$ & $39 / 69$ & $0.68(0.42-1.08)$ & 0.102 \\
\hline \multicolumn{9}{|l|}{ Pack-years } \\
\hline 0 & $298 / 305$ & $436 / 357$ & 1.40 (1.09-I.79) & 0.008 & 644/57I & $90 / 91$ & $0.76(0.53-1.10)$ & 0.143 \\
\hline$\leq 30$ & $102 / 182$ & $170 / 201$ & $1.74(1.19-2.54)$ & 0.004 & $248 / 331$ & $24 / 52$ & $0.56(0.31-1.01)$ & 0.053 \\
\hline$>30$ & $61 / 53$ & $74 / 75$ & $0.7 I(0.42-I .2 I)$ & 0.205 & $120 / 111$ & $15 / 17$ & $0.92(0.4 I-2.04)$ & 0.833 \\
\hline \multicolumn{9}{|l|}{ Drinking status } \\
\hline Never & $377 / 282$ & $556 / 318$ & $1.36(1.09-1.69)$ & 0.007 & $827 / 516$ & $106 / 84$ & $0.70(0.50-0.98)$ & 0.035 \\
\hline Ever & $84 / 258$ & $124 / 315$ & $1.28(0.88-1.86)$ & 0.201 & $185 / 497$ & $23 / 76$ & $0.79(0.45-1.4 I)$ & 0.429 \\
\hline \multicolumn{9}{|l|}{ Tumor site } \\
\hline Cardia & $102 / 540$ & $138 / 633$ & $1.36(0.99-1.87)$ & 0.059 & $212 / 1013$ & $28 / 160$ & $0.76(0.47-1.22)$ & 0.252 \\
\hline Non-cardia & $359 / 540$ & $542 / 633$ & $1.31(1.08-1.60)$ & 0.007 & $800 / 1013$ & $101 / 160$ & $0.72(0.53-0.97)$ & 0.030 \\
\hline \multicolumn{9}{|l|}{ TNM stage } \\
\hline $\mathrm{I} / \mathrm{II}$ & $184 / 540$ & $285 / 633$ & I.42 (I.I I-I.82) & 0.006 & $414 / 1013$ & $55 / 160$ & $0.74(0.5 \mathrm{I}-\mathrm{I} .07)$ & 0.112 \\
\hline III/IV & $277 / 540$ & $395 / 633$ & 1.28 (I.03-I.59) & 0.024 & $598 / 1013$ & $74 / 160$ & $0.72(0.52-1.00)$ & 0.050 \\
\hline
\end{tabular}

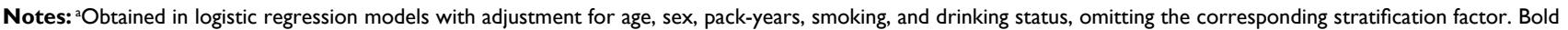
represents any values with a $95 \% \mathrm{Cl}$ excluding I or $P<0.05$.

Abbreviation: AOR, adjusted odds ratio.

Table 3 Stratification analysis of ERCC2 gene variant genotypes with gastric cancer risk

\begin{tabular}{|c|c|c|c|c|c|c|c|c|}
\hline \multirow[t]{2}{*}{ Variables } & \multicolumn{2}{|c|}{$\begin{array}{l}\text { rs3810366 } \\
\text { (cases/controls) }\end{array}$} & \multirow[t]{2}{*}{ AOR (95\% Cl) } & \multirow[t]{2}{*}{$P$-value ${ }^{a}$} & \multicolumn{2}{|c|}{$\begin{array}{l}\text { rs238406 } \\
\text { (cases/controls) }\end{array}$} & \multirow[t]{2}{*}{ AOR $(95 \% \mathrm{Cl})$} & \multirow[t]{2}{*}{$P$-value ${ }^{a}$} \\
\hline & $\mathrm{CC}$ & CG/GG & & & GG & GT/TT & & \\
\hline \multicolumn{9}{|c|}{ Median age, years } \\
\hline$\leq 58$ & $|73 / 33|$ & $425 / 685$ & $1.19(0.94-1.50)$ & 0.145 & $157 / 298$ & $44|/ 7| 8$ & I. 17 (0.92-I.48) & 0.196 \\
\hline$>58$ & $158 / 48$ & $385 / 109$ & $1.06(0.72-1.57)$ & 0.755 & $139 / 45$ & $404 / 112$ & $1.17(0.79-1.75)$ & 0.437 \\
\hline \multicolumn{9}{|l|}{ Gender } \\
\hline Males & $227 / 266$ & $522 / 523$ & $1.20(0.93-1.54)$ & 0.155 & $201 / 242$ & $548 / 547$ & $1.22(0.95-1.58)$ & 0127 \\
\hline Females & $104 / 113$ & 288/27I & $1.25(0.88-1.76)$ & 0.215 & $95 / 101$ & $297 / 283$ & I.24 (0.87-I.77) & 0.234 \\
\hline \multicolumn{9}{|l|}{ Smoking status } \\
\hline Never & $211 / 202$ & $523 / 460$ & $1.19(0.91-1.55)$ & 0.207 & $194 / 182$ & $540 / 480$ & $1.20(0.91-1.58)$ & 0.188 \\
\hline Ever & $120 / 177$ & $287 / 334$ & $1.27(0.92-1.75)$ & 0.142 & $102 / 161$ & $305 / 350$ & $1.32(0.95-1.83)$ & 0.103 \\
\hline \multicolumn{9}{|l|}{ Pack-years } \\
\hline 0 & $211 / 202$ & $523 / 460$ & $1.19(0.91-1.55)$ & 0.207 & $194 / 182$ & $540 / 480$ & $1.20(0.9 \mid-1.58)$ & 0.188 \\
\hline$\leq 30$ & $80 / 137$ & $192 / 246$ & $1.43(0.97-2.12)$ & 0.075 & $70 / 126$ & $202 / 257$ & $1.44(0.96-2.16)$ & 0.075 \\
\hline$>30$ & $40 / 40$ & $95 / 88$ & $\mathrm{I} .07(0.6 \mathrm{I}-\mathrm{I} .87)$ & 0.824 & $32 / 35$ & $103 / 93$ & $1.19(0.66-2.16)$ & 0.564 \\
\hline \multicolumn{9}{|l|}{ Drinking status } \\
\hline Never & $264 / 199$ & $669 / 401$ & $1.32(1.04-1.68)$ & 0.022 & $238 / 181$ & $695 / 419$ & $1.32(1.03-1.69)$ & 0.027 \\
\hline Ever & $67 / 180$ & $|4| / 393$ & $1.03(0.69-1.53)$ & 0.888 & $58 / 162$ & $|50 / 4| \mid$ & $1.13(0.74-1.69)$ & 0.602 \\
\hline \multicolumn{9}{|l|}{ Tumor site } \\
\hline Cardia & $75 / 379$ & $165 / 794$ & $1.19(0.85-1.67)$ & 0.318 & $67 / 343$ & $173 / 830$ & $1.20(0.84-1.70)$ & 0.318 \\
\hline Non-cardia & $256 / 379$ & $645 / 794$ & $1.19(0.97-1.48)$ & 0.103 & $229 / 343$ & $672 / 830$ & $1.21(0.97-1.50)$ & 0.093 \\
\hline \multicolumn{9}{|l|}{ TNM stage } \\
\hline $\mathrm{I} / \mathrm{II}$ & $143 / 379$ & $326 / 794$ & $1.18(0.9|-| .53)$ & 0.223 & $126 / 343$ & $343 / 830$ & $1.22(0.93-1.60)$ & 0.152 \\
\hline III/IV & $188 / 379$ & $484 / 794$ & $1.22(0.97-1.54)$ & 0.088 & $170 / 343$ & $502 / 830$ & $1.21(0.95-1.54)$ & 0.116 \\
\hline
\end{tabular}

Notes: ${ }^{\circ}$ Obtained in logistic regression models with adjustment for age, gender, pack-years, smoking, and drinking status, omitting the corresponding stratification factor. Bold represents any values with a $95 \% \mathrm{Cl}$ excluding I or $P<0.05$.

Abbreviation: AOR, adjusted odds ratio. 
genotypes of the rs2298881 C>A polymorphism were significantly correlated with decreased ERCC1 mRNA expression in Chinese subjects $(P=0.003)$, Africans $(P<0.0001)$, and combined subjects $(P<0.0001)$. However, no genotype expression correlation was found for the rs $3212986 \mathrm{C}>\mathrm{A}$ and rs11615 G>A polymorphisms in combined subjects.

\section{Discussion}

In the present hospital-based case-control study, we investigated the association between the polymorphisms in the NER genes and gastric cancer risk in a southern Chinese population. We observed a significantly increased gastric cancer risk associated with the ERCC1 rs2298881 A variant allele. However, we found that $E R C C 1$ rs3212986 A variant allele was associated with decreased risk of gastric cancer. We also confirmed that the ERCC1 rs $2298881 \mathrm{C}>\mathrm{A}$ polymorphism was associated with a decrease in ERCC1 mRNA expression. However, no association with gastric cancer risk was detected for the polymorphisms in the $X P A, X P D$, and $X P F$ genes. To the best of our knowledge, this is by far the most comprehensive study investigating the association between the NER pathway genes and gastric cancer risk.

ERCC1 gene is located on chromosome 19q32.32, consisting of 10 exons and encoding a 297 amino acid protein. The ERCC1 protein is an indispensable component of the NER pathway. ${ }^{37,38}$ It interacts with XPA, XPF, and/or RPA, and catalyzes the $5^{\prime}$ cleavage of DNA lesions. ${ }^{39}$ Given the critical role of ERCC1 protein in NER, it is biologically plausible that potentially functional ERCC1 gene variants could modify gastric cancer risk. Our findings are in accordance with others. For instance, He et al reported that ERCC1 rs11615 G>A was associated with an increased risk of breast cancer. ${ }^{40}$ Likewise, the ERCC1 rs11615 G>A polymorphism was shown to increase the risk of developing lung cancer. ${ }^{41} \mathrm{It}$ is worth mentioning that we previously observed that ERCC1 rs11615A and rs2298881C variant alleles were associated with increased gastric cancer risk in an eastern Chinese population. ${ }^{42}$ Moreover, patients with 2-3 $E R C C 1$ risk genotypes had a significantly increased risk of gastric cancer compared with those with 0-1 ERCC1 risk genotypes. ${ }^{42}$ However, the previous study did not detect an association between the rs3212986 polymorphism and gastric cancer risk. The discrepant results between the former study and the present study might be due to the different population selected. Our previous study population was recruited from East China, while the current study population was recruited from South China. Apart from our studies, two published studies regarding ERCC1 polymorphisms and gastric cancer risk were conducted in Italian population with relatively small sample sizes. ${ }^{43,44}$ One study included 314 cases and 548 controls, and the other included 126 cases and 144 controls. No significant association was detected in these two studies. However, all the included polymorphisms of ERCC1 in these two studies were not under investigation in the present study.

In the stratification analysis, our data suggested that the risk effect of ERCC1 rs2298881 CA/AA genotypes remained significant in males, never-smokers, pack-year of 0 , packyears $\leq 30$, never drinkers, non-cardia, stage I/II, and stage III/IV subgroups. The association between decreased gastric cancer risk and ERCC1 rs3212986 was more evident in subgroups of median age $\leq 58$ years, males, never-drinkers, and non-cardia tumor. This phenomenon can be explained by the concept that susceptible individuals are likely to have a light exposure to risk factors. Young individuals, never smokers, or never drinkers are tended to be exposed to less environmental carcinogens. Thus, the role of genetic variants might not be outweighed by carcinogens in carcinogenesis in such subgroups. Considering the reduced sample sizes in the stratification analysis, some results might be just chance findings. Therefore, these results should be interpreted with caution. We further adopted the public data on ERCC1 genotypes and mRNA levels for the genotype-phenotype association analysis. A significant correlation between ERCC1 mRNA levels and rs2298881 C>A genotypes was observed, which provide further evidence that rs2298881 $\mathrm{C}>\mathrm{A}$ may associate with gastric cancer by mRNA expression alteration, sequentially DNA repair capacity alteration. Therefore, additional larger case-control studies with functional analysis are warranted to explore the exact role of $E R C C 1$ in gastric cancer risk.

We failed to detect any relationship between other polymorphisms and gastric cancer risk. Lack of an association of gastric cancer susceptibility with single NER pathway gene variants was also reported by other studies. For instance, in a case-control study including 246 cases and 1175 controls, no significant association was observed between the analyzed polymorphisms in the $M S H 2, M L H 1, X R C C 1, O G G 1$, and ERCC2 genes and gastric cancer risk. ${ }^{45}$ However, some previous studies have demonstrated that some polymorphisms including rs11615 G>A were independent risk factors for gastric cancer. ${ }^{42}$ Such a discrepancy among studies might be partly due to the limited sample sizes; small sample studies may not have sufficient statistical power to reveal an association. Another possible explanation was that the effect of each single variant was too weak to be detected. Moreover, the potential effect of polymorphisms in gastric cancer risk 
may be dissimulated by other complex exposures or environmental-genetic interactions.

Although we extensively analyzed a number of polymorphisms in the NER core pathway genes, some limitations still existed in this study. First, due to the nature of a retrospective study, selection bias and recall bias could not be completely avoided. To minimize such biases, we further performed multivariate logistic regression analysis on potential confounding factors such as age, smoking, and drinking status. Second, gastric cancer is a heterogeneous disease affected by multiple factors including $H$. pylori infection, environmental exposures, and diet habits, yet these data were not available for further analysis. Third, the sample size in the subgroup analysis was relatively small, which might limit the statistical power in the stratification analysis. Fourth, we adopted only the public data to preliminarily investigate the correlation between ERCC1 genotype and mRNA expression. The findings should be validated in gastric tissues in the future. We failed to quantify the ERCC1 mRNA levels in the target tissue of the included subjects due to tissue access constraints. Finally, as all participants were recruited from a hospital in South China, special caution should be paid in extrapolating the results to other populations.

In conclusion, we found that the ERCC1 gene rs2298881 $\mathrm{C}>\mathrm{A}$ and rs3212986 C>A polymorphisms were associated with gastric cancer susceptibility in a southern Chinese population. Well-designed studies with larger sample sizes and functional analysis are required to further verify our findings.

\section{Acknowledgments}

This study was supported by grants from National Natural Science Foundation of China (Grant No. 81502046), the Natural Science Foundation of Guangdong Province (Grant No. 2015A030310324), and the National Science Fund for Distinguished Young Scholars (Grant No. 81325018).

\section{Disclosure}

The authors report no conflicts of interest in this work.

\section{References}

1. Ferlay J, Soerjomataram I, Dikshit R, et al. Cancer incidence and mortality worldwide: sources, methods and major patterns in GLOBOCAN 2012. Int J Cancer. 2015;136(5):E359-E386.

2. Torre LA, Siegel RL, Ward EM, Jemal A. Global cancer incidence and mortality rates and trends-an update. Cancer Epidemiol Biomarkers Prev. 2016;25(1):16-27.

3. Chen W, Zheng R, Baade PD, et al. Cancer statistics in China, 2015. CA Cancer J Clin. 2016;66(2):115-132.

4. Amedei A, Benagiano M, della Bella C, Niccolai E, D'Elios MM. Novel immunotherapeutic strategies of gastric cancer treatment. $J$ Biomed Biotechnol. 2011;2011:437348.
5. Ajani JA, Lee J, Sano T, Janjigian YY, Fan D, Song S. Gastric adenocarcinoma. Nat Rev Dis Primers. 2017;3:17036.

6. Vogiatzi P, Vindigni C, Roviello F, Renieri A, Giordano A. Deciphering the underlying genetic and epigenetic events leading to gastric carcinogenesis. J Cell Physiol. 2007;211(2):287-295.

7. Ye W, Held M, Lagergren J, et al. Helicobacter pylori infection and gastric atrophy: risk of adenocarcinoma and squamous-cell carcinoma of the esophagus and adenocarcinoma of the gastric cardia. J Natl Cancer Inst. 2004;96(5):388-396.

8. Ishaq S, Nunn L. Helicobacter pylori and gastric cancer: a state of the art review. Gastroenterol Hepatol Bed Bench. 2015;8(Suppl 1):S6-S14.

9. Ladeiras-Lopes R, Pereira AK, Nogueira A, et al. Smoking and gastric cancer: systematic review and meta-analysis of cohort studies. Cancer Causes Control. 2008;19(7):689-701.

10. Palli D. Epidemiology of gastric cancer: an evaluation of available evidence. J Gastroenterol. 2000;35(Suppl 12):84-89.

11. Tran GD, Sun XD, Abnet CC, et al. Prospective study of risk factors for esophageal and gastric cancers in the Linxian general population trial cohort in China. Int J Cancer. 2005;113(3):456-463.

12. Yan C, Zhu M, Huang T, Yu F, Jin G. Genome-wide association studies identified loci contribute to phenotypic variance of gastric cancer. Gut. Epub 2017 Sep 22.

13. Ye XS, Yu C, Aggarwal A, Reinhard C. Genomic alterations and molecular subtypes of gastric cancers in Asians. Chin J Cancer. 2016;35:42.

14. Wood RD, Mitchell M, Sgouros J, Lindahl T. Human DNA repair genes. Science. 2001;291(5507):1284-1289.

15. Hoeijmakers JH. Genome maintenance mechanisms for preventing cancer. Nature. 2001;411(6835):366-374.

16. Gillet LC, Scharer OD. Molecular mechanisms of mammalian global genome nucleotide excision repair. Chem Rev. 2006;106(2):253-276.

17. Ishikawa T, Zhang SS, Qin X, et al. DNA repair and cancer: lessons from mutant mouse models. Cancer Sci. 2004;95(2):112-117.

18. Cordonnier AM, Fuchs RP. Replication of damaged DNA: molecular defect in xeroderma pigmentosum variant cells. Mutat Res. 1999;435(2):111-119.

19. Nouspikel T. DNA repair in mammalian cells: nucleotide excision repair: variations on versatility. Cell Mol Life Sci. 2009;66(6):994-1009.

20. Cleaver JE. Common pathways for ultraviolet skin carcinogenesis in the repair and replication defective groups of xeroderma pigmentosum. J Dermatol Sci. 2000;23(1):1-11.

21. Araki M, Masutani C, Takemura M, et al. Centrosome protein centrin $2 /$ caltractin 1 is part of the xeroderma pigmentosum group $\mathrm{C}$ complex that initiates global genome nucleotide excision repair. $J$ Biol Chem. 2001;276(22):18665-18672.

22. Sugasawa K. Multiple DNA damage recognition factors involved in mammalian nucleotide excision repair. Biochemistry (Mosc). 2011;76(1):16-23.

23. Coin F, Oksenych V, Egly JM. Distinct roles for the XPB/p52 and XPD/ p44 subcomplexes of TFIIH in damaged DNA opening during nucleotide excision repair. Mol Cell. 2007;26(2):245-256.

24. Evans E, Moggs JG, Hwang JR, Egly JM, Wood RD. Mechanism of open complex and dual incision formation by human nucleotide excision repair factors. EMBO J. 1997;16(21):6559-6573.

25. Matsunaga T, Mu D, Park CH, Reardon JT, Sancar A. Human DNA repair excision nuclease. Analysis of the roles of the subunits involved in dual incisions by using anti-XPG and anti-ERCC1 antibodies. J Biol Chem. 1995;270(35):20862-20869.

26. Enzlin JH, Scharer OD. The active site of the DNA repair endonuclease XPF-ERCC1 forms a highly conserved nuclease motif. EMBO J. 2002;21(8):2045-2053.

27. Liu J, Sun L, Xu Q, et al. Association of nucleotide excision repair pathway gene polymorphisms with gastric cancer and atrophic gastritis risks. Oncotarget. 2016;7(6):6972-6983.

28. Wei ZH, Guo WH, Wu J, Suo WH, Fu GH. A nonsense mutation in the Xeroderma pigmentosum complementation group $\mathrm{F}(\mathrm{XPF})$ gene is associated with gastric carcinogenesis. Gene. 2014;537(2):238-244.

29. Hua RX, Zhuo ZJ, Shen GP, et al. Polymorphisms in the XPC gene and gastric cancer susceptibility in a Southern Chinese population. Onco Targets Ther. 2016;9:5513-5519. 
30. Hua RX, Zhuo ZJ, Zhu J, et al. Association between genetic variants in the XPG gene and gastric cancer risk in a Southern Chinese population. Aging (Albany NY). 2016;8(12):3311-3320.

31. He J, Qiu LX, Wang MY, et al. Polymorphisms in the XPG gene and risk of gastric cancer in Chinese populations. Hum Genet. 2012;131(7): 1235-1244.

32. He J, Zhong W, Zeng J, et al. LMO1 gene polymorphisms contribute to decreased neuroblastoma susceptibility in a Southern Chinese population. Oncotarget. 2016;7(16):22770-22778.

33. Li J, Zou L, Zhou Y, et al. A low-frequency variant in SMAD7 modulates TGF-beta signaling and confers risk for colorectal cancer in Chinese population. Mol Carcinog. 2017;56(7):1798-1807.

34. Lou J, Gong J, Ke J, et al. A functional polymorphism located at transcription factor binding sites, rs6695837 near LAMC1 gene, confers risk of colorectal cancer in Chinese populations. Carcinogenesis. 2017;38(2):177-183.

35. He J, Shi TY, Zhu ML, Wang MY, Li QX, Wei QY. Associations of Lys939Gln and Ala499Val polymorphisms of the XPC gene with cancer susceptibility: a meta-analysis. Int J Cancer. 2013;133(8):1765-1775.

36. Washington $\mathrm{K}$. 7th edition of the AJCC cancer staging manual: stomach. Ann Surg Oncol. 2010;17(12):3077-3079.

37. van Duin M, de Wit J, Odijk H, et al. Molecular characterization of the human excision repair gene ERCC-1: cDNA cloning and amino acid homology with the yeast DNA repair gene RAD10. Cell. 1986;44(6):913-923.
38. Smith AH, Spangler WL, Burton RR, Rhode EA. Responses of domestic fowl to repeated $+\mathrm{Gz}$ acceleration. Aviat Space Environ Med. 1979;50(11):1134-1138.

39. Friedberg EC. DNA damage and repair. Nature. 2003;421(6921): 436-440.

40. He BS, Xu T, Pan YQ, et al. Nucleotide excision repair pathway gene polymorphisms are linked to breast cancer risk in a Chinese population. Oncotarget. 2016;7(51):84872-84882.

41. Deng Q, Sheng L, Su D, et al. Genetic polymorphisms in ATM, ERCC1, APE1 and iASPP genes and lung cancer risk in a population of southeast China. Med Oncol. 2011;28(3):667-672.

42. He J, Xu Y, Qiu LX, et al. Polymorphisms in ERCC1 and XPF genes and risk of gastric cancer in an eastern Chinese population. PLoS One. 2012;7(11):e49308.

43. Palli D, Polidoro S, D'Errico M, et al. Polymorphic DNA repair and metabolic genes: a multigenic study on gastric cancer. Mutagenesis. 2010;25(6):569-575.

44. Ruzzo A, Canestrari E, Maltese P, et al. Polymorphisms in genes involved in DNA repair and metabolism of xenobiotics in individual susceptibility to sporadic diffuse gastric cancer. Clin Chem Lab Med. 2007;45(7):822-828.

45. Capella G, Pera G, Sala N, et al. DNA repair polymorphisms and the risk of stomach adenocarcinoma and severe chronic gastritis in the EPIC-EURGAST study. Int J Epidemiol. 2008;37(6):1316-1325. 


\section{Supplementary materials}

Table SI Clinical and demographic characteristics of gastric cancer cases and cancer-free controls

\begin{tabular}{|c|c|c|c|}
\hline Variables & No. of cases (\%) & No. of controls (\%) & $P$-value ${ }^{a}$ \\
\hline All subjects & $1142(100.0)$ & $1173(100.0)$ & \\
\hline \multicolumn{4}{|l|}{ Gender } \\
\hline Male & $749(65.6)$ & $789(67.3)$ & 0.393 \\
\hline Female & $393(34.4)$ & $384(32.7)$ & \\
\hline Age, years & $15-86$ & $16-80$ & \\
\hline Mean \pm SD & $56.3 \pm 12.5$ & $45.2 \pm 11.6$ & $<0.0001$ \\
\hline$\leq 50$ & $334(29.3)$ & $789(67.3)$ & \\
\hline $51-60$ & $362(3 \mid .7)$ & $285(24.3)$ & \\
\hline $6 I-70$ & $312(27.3)$ & $73(6.2)$ & \\
\hline$>70$ & $134(\mid 1.7)$ & $26(2.2)$ & \\
\hline \multicolumn{4}{|l|}{ Smoking status } \\
\hline Never & $735(64.4)$ & $662(56.4)$ & $<0.0001$ \\
\hline Ever & $407(35.6)$ & $5 I 1(43.6)$ & \\
\hline \multicolumn{4}{|l|}{ Drinking status } \\
\hline No & $934(81.8)$ & $600(5 \mid .2)$ & $<0.0001$ \\
\hline Yes & $208(18.2)$ & $573(48.8)$ & \\
\hline \multicolumn{4}{|l|}{ Pack-years } \\
\hline 0 & $735(64.4)$ & $662(56.4)$ & $<0.0001$ \\
\hline$\leq 30$ & $272(23.8)$ & $383(32.7)$ & \\
\hline$>30$ & $135(11.8)$ & $128(10.9)$ & \\
\hline \multicolumn{4}{|l|}{ Sites } \\
\hline Cardia & $240(21.0)$ & - & \\
\hline Non-cardia & $902(79.0)$ & - & \\
\hline \multicolumn{4}{|l|}{ TNM stages } \\
\hline I & $140(12.3)$ & - & \\
\hline ॥ & $329(28.8)$ & - & \\
\hline III & $456(39.9)$ & - & \\
\hline IV & $217(19.0)$ & - & \\
\hline
\end{tabular}


Table S2 ERCCI mRNA expression by the genotypes of polymorphisms, using data from the HapMapa

\begin{tabular}{|c|c|c|c|c|c|c|c|c|c|c|c|c|}
\hline \multirow[t]{2}{*}{ Population } & \multicolumn{4}{|c|}{ mRNA expression (rs229888I) } & \multicolumn{4}{|c|}{ mRNA expression (rs32 I 2986) } & \multicolumn{4}{|c|}{ mRNA expression (rs I |6I5) } \\
\hline & Genotypes & No. & Mean \pm SD & $P$-value ${ }^{b}$ & Genotypes & No. & Mean \pm SD & $P$-value ${ }^{b}$ & Genotypes & No. & Mean \pm SD & $P$-value ${ }^{b}$ \\
\hline \multirow[t]{4}{*}{$\mathrm{CHB}$} & $\mathrm{CC}$ & 15 & $6.8 I \pm 0.08$ & $0.003^{c}$ & $\mathrm{CC}$ & 20 & $6.74 \pm 0.13$ & $0.442^{c}$ & GG & 29 & $6.73 \pm 0.11$ & $0.044^{c}$ \\
\hline & $A C$ & 20 & $6.76 \pm 0.09$ & 0.126 & $A C$ & 19 & $6.77 \pm 0.09$ & 0.416 & AG & 12 & $6.79 \pm 0.10$ & 0.144 \\
\hline & AA & 10 & $6.68 \pm 0.13$ & 0.006 & AA & 5 & $6.77 \pm 0.07$ & 0.664 & $\mathrm{AA}$ & 4 & $6.83 \pm 0.07$ & 0.111 \\
\hline & AC/AA & 30 & $6.73 \pm 0.11$ & 0.026 & AC/AA & 24 & $6.77 \pm 0.08$ & $0.377^{d}$ & AG/AA & 16 & $6.80 \pm 0.09$ & 0.054 \\
\hline \multirow[t]{4}{*}{ JPT } & $\mathrm{CC}$ & 9 & $6.8 \mathrm{I} \pm 0.07$ & $0.242^{c}$ & $\mathrm{CC}$ & 31 & $6.75 \pm 0.09$ & $0.442^{c}$ & GG & 21 & $6.75 \pm 0.10$ & $0.872^{c}$ \\
\hline & $A C$ & 26 & $6.74 \pm 0.11$ & 0.067 & $A C$ & 13 & $6.77 \pm 0.12$ & 0.442 & AG & 22 & $6.76 \pm 0.10$ & 0.846 \\
\hline & AA & 10 & $6.76 \pm 0.08$ & 0.118 & AA & 0 & - & - & AA & 2 & $6.76 \pm 0.06$ & 0.976 \\
\hline & AC/AA & 36 & $6.74 \pm 0.10$ & 0.060 & $A C / A A$ & 13 & $6.77 \pm 0.12$ & $0.442^{\mathrm{d}}$ & $A G / A A$ & 24 & $6.76 \pm 0.10$ & 0.848 \\
\hline \multirow[t]{4}{*}{ CEU } & $\mathrm{CC}$ & 79 & $6.77 \pm 0.12$ & $0.370^{c}$ & $\mathrm{CC}$ & 52 & $6.77 \pm 0.13$ & $0.725^{c}$ & GG & 6 & $6.85 \pm 0.13$ & $0.447^{c}$ \\
\hline & $A C$ & II & $6.74 \pm 0.18$ & 0.370 & $A C$ & 35 & $6.74 \pm 0.12$ & 0.279 & AG & 49 & $6.76 \pm 0.14$ & 0.168 \\
\hline & AA & 0 & - & - & $\mathrm{AA}$ & 3 & $6.95 \pm 0.04$ & 0.026 & $\mathrm{AA}$ & 35 & $6.77 \pm 0.11$ & 0.111 \\
\hline & AC/AA & II & $6.74 \pm 0.18$ & 0.370 & $A C / A A$ & 38 & $6.76 \pm 0.13$ & 0.620 & $A G / A A$ & 84 & $6.76 \pm 0.13$ & 0.129 \\
\hline \multirow[t]{4}{*}{ YRI } & $\mathrm{CC}$ & 76 & $6.80 \pm 0.09$ & $<0.000 I^{c}$ & $\mathrm{CC}$ & 39 & $6.77 \pm 0.10$ & $0.208^{c}$ & GG & 87 & $6.79 \pm 0.10$ & $0.137^{c}$ \\
\hline & $A C$ & II & $6.7 I \pm 0.07$ & 0.002 & $A C$ & 45 & $6.8 \mathrm{I} \pm 0.09$ & 0.046 & AG & 3 & $6.7 I \pm 0.05$ & 0.137 \\
\hline & AA & 2 & $6.6 \mathrm{I} \pm 0.003$ & 0.004 & AA & 6 & $6.76 \pm 0.05$ & 0.976 & AA & 0 & - & - \\
\hline & $A C / A A$ & 13 & $6.70 \pm 0.07$ & $0.000 I^{d}$ & $A C / A A$ & 51 & $6.80 \pm 0.09$ & 0.065 & $A G / A A$ & 3 & $6.7 \mathrm{I} \pm 0.05$ & 0.137 \\
\hline \multirow[t]{4}{*}{ All } & $\mathrm{CC}$ & 179 & $6.79 \pm 0.10$ & $<0.000 I^{c}$ & $\mathrm{CC}$ & 142 & $6.76 \pm 0.11$ & $0.095^{c}$ & GG & 143 & $6.78 \pm 0.10$ & $0.599^{c}$ \\
\hline & $A C$ & 68 & $6.74 \pm 0.11$ & 0.001 & $A C$ & 112 & $6.78 \pm 0.11$ & 0.243 & AG & 86 & $6.76 \pm 0.12$ & 0.385 \\
\hline & AA & 22 & $6.71 \pm 0.11$ & 0.001 & AA & 14 & $6.80 \pm 0.09$ & 0.162 & AA & $4 I$ & $6.77 \pm 0.10$ & 0.793 \\
\hline & AC/AA & 90 & $6.73 \pm 0.11$ & $<0.000 \mathrm{I}^{\mathrm{d}}$ & $A C / A A$ & 126 & $6.78 \pm 0.10$ & $0.149^{d}$ & $A G / A A$ & 127 & $6.77 \pm 0.12$ & 0.435 \\
\hline
\end{tabular}

Notes: ${ }^{E} E R C C I$ genotyping data and mRNA expression levels for ERCCI by genotypes were obtained from the HapMap Phase II release 23 data from EBV-transformed lymphoblastoid cell lines from 270 individuals, including 45 unrelated CHB. ${ }^{b}$ Two-sided Student's $t$-test within the stratum. ${ }^{C P}$-values for the trend test of ERCCI mRNA expression among three genotypes for each polymorphism from a general linear model. ${ }^{\mathrm{d}}$ There were missing data because genotyping data were not available. Bold represents any values $P<0.05$.

Abbreviations: CEU, Utah residents with ancestry from northern and western Europe; CHB, Han Chinese in Beijing, China; JPT, Japanese in Tokyo; YRI, Yoruba in Ibadan, Nigeria.

Cancer Management and Research

\section{Publish your work in this journal}

Cancer Management and Research is an international, peer-reviewed open access journal focusing on cancer research and the optimal use of preventative and integrated treatment interventions to achieve improved outcomes, enhanced survival and quality of life for the cancer patient. The manuscript management system is completely online and includes

\section{Dovepress}

a very quick and fair peer-review system, which is all easy to use. Visit $\mathrm{http}: / / \mathrm{www}$.dovepress.com/testimonials.php to read real quotes from published authors. 